\title{
Pulsed thermography: a useful tool to determine porosity in composite materials ?
}

\author{
by V. Feuillet*, N. Dujardin*, M. Fois*, L. Ibos*, C. Poilâne**, Y. Candau*
}

* Université Paris-Est, CERTES, EA 3481, Créteil, France

** Université de Caen Basse-Normandie / CNRS / CEA / ENSICAEN, CIMAP, UMR 6252, Caen, France

** Université du Maine, LAUM, UMR 6613, Le Mans, France

\begin{abstract}
In this study, active infrared thermography is used to investigate porosity in flax/epoxy composites. An experimental set-up is developed using pulsed thermography in reflection or transmission modes coupled with transient heat transfer modeling. An inverse analysis and a calibration procedure are carried out to estimate both spatial thermal diffusivity and conductivity distributions of the studied sample. Sensitivity study and estimation results show that rear face measurements are more suitable than front face measurements to characterize the sample. A comparison of the porosity rates of the studied samples and the estimated thermal diffusivity distributions is eventually performed.
\end{abstract}

\section{Introduction}

The presence of porosity in composite materials is inherent in the manufacturing process. The porosity rate is directly related to poor mechanical performance of the material. This is why low levels of porosity are required to ensure the performance of composites [1, 2]. In aeronautics, for example, only $2 \%$ porosity rates are accepted in some cases [3]. Porosity can be measured by a considerable number of destructive or non-destructive techniques, such as ultrasonic attenuation, acid digestion or X-ray tomography [4]. The most common technique (ultrasonic method) can provide an estimate, however with a consequent uncertainty (25\%, even more, [5]). We propose to study the porosity of materials by a non destructive technique: pulsed thermography.

Infrared thermography is an alternative to other techniques of conventional nondestructive testing commonly used to study a wide range of materials. It has several advantages over other techniques, including the opportunity to study a sample surface without substantial scanning time. This technique consists in applying a thermal excitation on one side of the sample and recording with an infrared camera temperature variation over time. Any defect, such as a cavity or an inhomogeneous density, will result in a change of the temperature distribution. This technique is particularly suitable for composite materials because their low thermal diffusivity produces a slow thermal response easier to record.

The use of infrared thermography to detect internal defects is relatively well documented [6-9]. The traditional thermographic approach uses laser or flash lamp to heat the sample. The spatial distribution of temperature in the moments following the excitation is used to trace the defects. Studies show a correlation between thermal diffusivity and the porosity of a composite. Thus, Connolly was able to demonstrate by active thermography in transmission mode that defects and porosity affect the apparent thermal diffusivity and reduce the heat flux on the rear face [8]. He also showed that the temperature reached on the rear face is lower for materials with high porosity.

In this paper natural fibers reinforced composites are investigated. The experimental approach is first described and the heat transfer modeling is then presented. Analysis of heat transfer in the sample leads to several levels of model complexity. The measurement analyzis shows how differences in diffusivity are a consequence of changes in porosity within the samples.

\section{Experimental setup}

In this work, pulsed thermography is used to characterize thermophysical properties of flax/epoxy composites. This kind of composite material is increasingly used in many fields such as transport, automotive, leisure, where high stiffness and low weight are required. Flax fibers are peculiarly interesting since they have specific mechanical properties comparable to glass fibers [10].

Flax/epoxy composite plates were prepared by contact-molding and provided by the laboratory of research on properties of new materials (LRPMN). One side of the plates is submitted to a heat pulse provided by four BALCAR ${ }^{\mathrm{TM}}$ flashes (3200J each). Temperature evolution on the stimulated face or the opposite face is recorded and analyzed. One long-wave IRFPA camera (FLIRTM SC7300 camera with a cooled detector) is used to acquire thermal images during

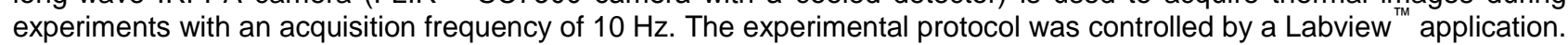
The investigated system is constituted by nine flax/epoxy composite samples presenting different porosity rates. The experimental setup is presented in figure 1 along with an example of recorded thermogram over the mosaic of nine composite samples. For ulterior processing, thermograms are averaged on a 5 pixels $\times 5$ pixels grid. 

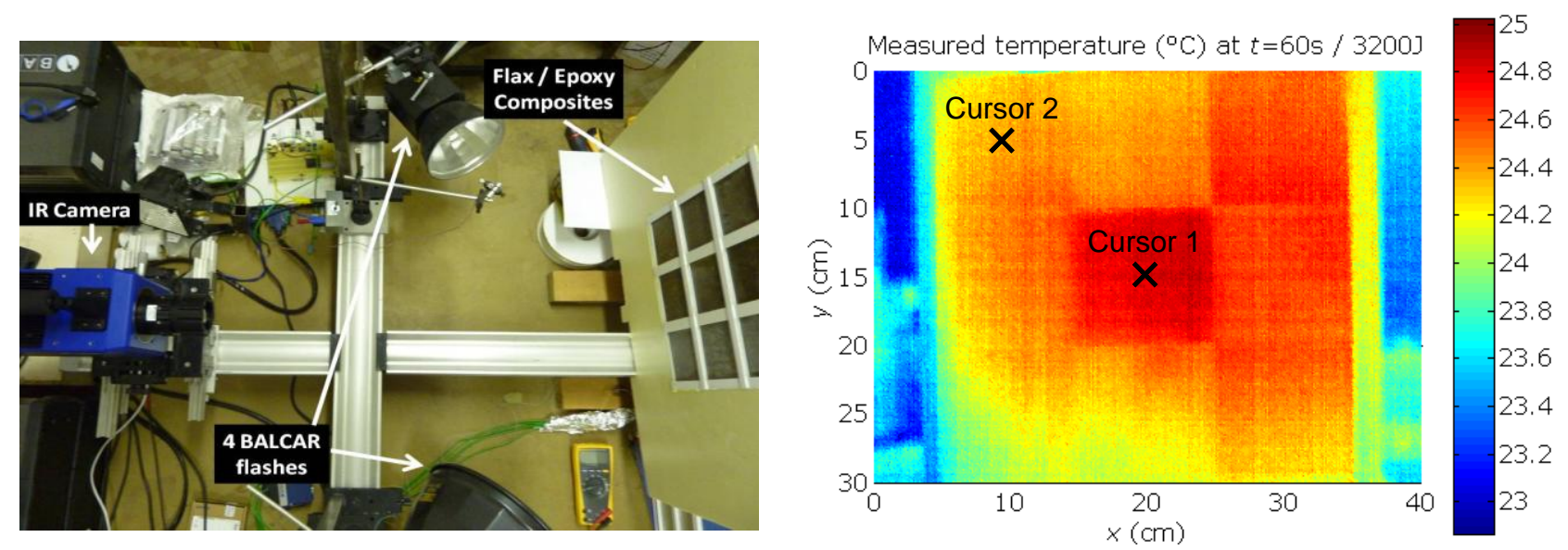

Fig. 1. Experimental setup and example of recorded thermogram over the mosaic of composite samples (samples are in the form of plate $10 \times 10 \mathrm{~cm}^{2}$ )

\section{Heat transfer modeling}

\subsection{Heat transfer problem solution}

In order to implement an inverse procedure to estimate parameters of interest, thermal models based on analytical solution of the heat equation are developed. Three different accuracy levels are proposed.

We consider each composite sample as a parallelepiped plate whose thickness is denoted e, thermal conductivity $k$ and thermal diffusivity $a$ (figure 2 ). The sample is submitted to heat exchanges both on front and rear faces (respectively global heat transfer coefficients $h_{0}$ and $h_{\mathrm{e}}$ ). In the case of an homogeneous absorbed heat energy per unit area $W$ at $t=0$ on the front face and since the thickness $e$ of the sample (between 2.2 and $4.0 \mathrm{~mm}$ depending on the sample) is very small compared to its lateral lengths $\left(10 \times 10 \mathrm{~cm}^{2}\right)$, the diffusion in the plane of the composite plate is neglected and heat transfer inside the sample is considered $1 D$ in the $z$-direction. In that case the heat equation inside the sample during heating and cooling is given as:

$$
\frac{1}{a} \frac{\partial T}{\partial t}=\frac{\partial^{2} T}{\partial z^{2}}
$$

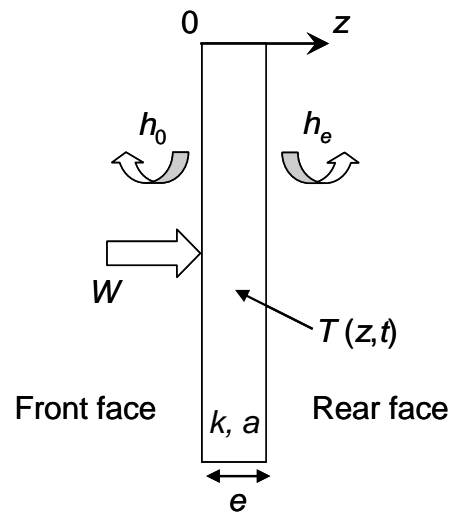

Fig. 2. Schematic view of the heat transfer modeling 
The first accuracy level of thermal modeling consists in neglecting heat exchange on both front and rear faces. Then solution of Eq. (1) by considering boundary conditions and using the integral transforms method is written as follows [11]:

$$
\Delta T(z, t)=\Gamma\left[1+2 \sum_{n=1}^{\infty} \cos (n \pi \delta) \exp \left(-\frac{n^{2} \pi^{2} t}{\tau}\right)\right]
$$

with $\Delta T$ the variation $T(z, t)-T(z, t=0)$ and :

$$
\Gamma=\frac{W a}{k e} \quad \tau=\frac{e^{2}}{a} \quad \delta=\frac{z}{e}
$$

where $\Gamma$ represents the final overheating of the sample with $h_{0}=h_{\mathrm{e}}=0$ (in ${ }^{\circ} \mathrm{C}$ ) and $\tau$ the diffusion time constant of the sample (in s). One should note that the adimensional parameter $\delta$ is equal to 0 and 1 respectively on front $(z=0)$ and rear $(z=e)$ faces of the sample.

The second accuracy level of thermal modeling consists in taking into account only the heat exchange on the front face, which leads to the following solution [11]:

$$
\Delta T(z, t)=\frac{2 \Gamma}{B_{0}} \sum_{n=1}^{\infty} \mu_{n}^{2} \frac{\sin \mu_{n} \cos \left[\mu_{n}(1-\delta)\right]}{\mu_{n}+\sin \mu_{n} \cos \mu_{n}} \exp \left(-\frac{\mu_{n}^{2} t}{\tau}\right)
$$

where $B_{0}=h_{0} e / k$ is the Biot number at the front face and $\mu_{n}$ are the positive roots of $\mu_{n} \tan \mu_{n}=B_{0}$.

Finally the third accuracy level of thermal modeling consists in taking into account heat exchanges on both front and rear faces to obtain [12]:

$$
\Delta T(z, t)=2 \Gamma \sum_{n=1}^{\infty} \frac{\xi_{n}\left[\xi_{n} \cos \left(\xi_{n} \delta\right)+B_{0} \sin \left(\xi_{n} \delta\right)\right]}{\left(\xi_{n}^{2}+B_{0}^{2}\right)\left(1+\frac{B_{e}}{\xi_{n}^{2}+B_{e}^{2}}\right)+B_{0}} \exp \left(-\frac{\xi_{n}^{2} t}{\tau}\right)
$$

where $B_{e}=h_{e} e / k$ is the Biot number on rear face and $\xi_{n}$ are the positive roots of $\left(\xi_{n}^{2}-B_{0} B_{e}\right) \tan \xi_{n}=\left(B_{0}+B_{e}\right) \xi_{n}$.

\subsection{Inverse procedure and statistical analysis}

In the case of the third accuracy level of thermal modeling, the unknown parameters to estimate from temperature measurements and inverse procedure are $\Gamma, \tau, B_{0}$ and $B_{e}$. The corresponding inverse problem is formulated in the least-squares sense and consists in finding the optimal solution that minimizes the functional:

$$
S(\beta)=\sum_{j=1}^{J}\left[T_{\text {meas }, j}-T_{e s t i m, j}(\beta)\right]^{2}
$$

where $T_{\text {meas }}$ are the measured temperatures, $T_{\text {estim }}$ are the estimated temperatures computed thanks to the heat transfer modeling presented in 3.1 with $\beta$ the vector of parameters to estimate, $J$ is the number of experimental data. $\beta$ elements are given as follows:

$$
\beta=\left[\Gamma ; \tau ; B_{0} ; B_{e}\right]
$$

Minimization of $S$ is performed iteratively by starting from an initial set $\beta_{\text {init }}$ which is refined progressively by using Levenberg-Marquardt algorithm [13] in order to estimate the final set $\hat{\beta}$. This minimization procedure requires to carry out a statistical analysis to determine the accuracy of the solution. Approximate confidence bounds for the estimated parameters are obtained thanks to a few assumptions and simplifications [14]. The variance-covariance matrix of the estimated parameter vector $\hat{\beta}$ can be approximated as: 


$$
\operatorname{cov}(\hat{\beta})=\sigma^{2}\left(m \cdot m^{t}\right)^{-1}
$$

where $m=\left(\partial T_{\text {estim }} / \partial \hat{\beta}\right)$ represents the Jacobian matrix and $m^{t}$ the transpose Jacobian matrix, $\sigma$ represents the standard deviation of the measurement estimated by:

$$
\sigma=\sqrt{\frac{S(\hat{\beta})}{J-q}}
$$

where $q$ is the number of estimated parameters. Finally the variance-covariance matrix is:

$$
\operatorname{cov}(\hat{\beta})=\left[\begin{array}{cccc}
\sigma_{\Gamma}^{2} & \sigma_{\Gamma \tau} & \sigma_{\Gamma B_{0}} & \sigma_{\Gamma B_{e}} \\
\sigma_{\tau \Gamma} & \sigma_{\tau}^{2} & \sigma_{\tau B_{0}} & \sigma_{\tau B_{e}} \\
\sigma_{B_{0} \Gamma} & \sigma_{B_{0} \tau} & \sigma_{B_{0}}^{2} & \sigma_{B_{0} B_{e}} \\
\sigma_{B_{e} \Gamma} & \sigma_{B_{e} \tau} & \sigma_{B_{e} B_{0}} & \sigma_{B_{e}}^{2}
\end{array}\right]
$$

We assume a normal distribution for the measurement errors. The statistical confidence bounds for estimated parameters $\beta_{i}$ are $\beta_{i}=\beta_{i} \pm 2 \sigma_{\beta_{i}}$ at a confidence level of $95 \%$ [15].

\section{Results}

\subsection{Sensitivity study}

The sensitivity study provides information about the influence of the experimental configuration (front or rear face measurements) on the estimation quality. Reduced sensitivity coefficients $C_{i}$ are expressed by:

$$
C_{i}=\beta_{i} \frac{\delta T_{\text {estim }}}{\delta \beta_{i}}
$$

where $\beta_{i}$ are the parameters to estimate (see Eq. (7)), $\delta T_{\text {estim }}$ and $\delta \beta_{i}$ refer to very small variations respectively of the estimated temperatures $T_{\text {estim }}$ and the parameter $\beta_{i}$.

The profiles in figure 3(a) refer to reduced sensitivity coefficients computed for a front face experiment by taking into account heat exchange on the front face for the sample located at the centre of the mosaic. They show that the two parameters $\tau$ and $\Gamma$ to estimate seem correlated since sensitivity curves present similar shapes. In addition the coefficients associated with the Biot number $B_{0}$ remain low throughout the experiment. However the same profiles for a rear face experiment exhibit curves with quite different shapes (figure 3(b)). So it might be easier to characterize correctly the sample from rear face measurements than from front face measurements. These observations are confirmed by presenting correlation coefficient $\sigma_{\Gamma \tau}$ distributions computed along the sample surface for each experiment. Front face measurements lead to correlation coefficients close to -1 (figure $3(\mathrm{c})$ ) while rear face measurements lead to values around 0.5 (figure $3(d)$ ) which is much more convenient to develop an inverse procedure. 
(a)

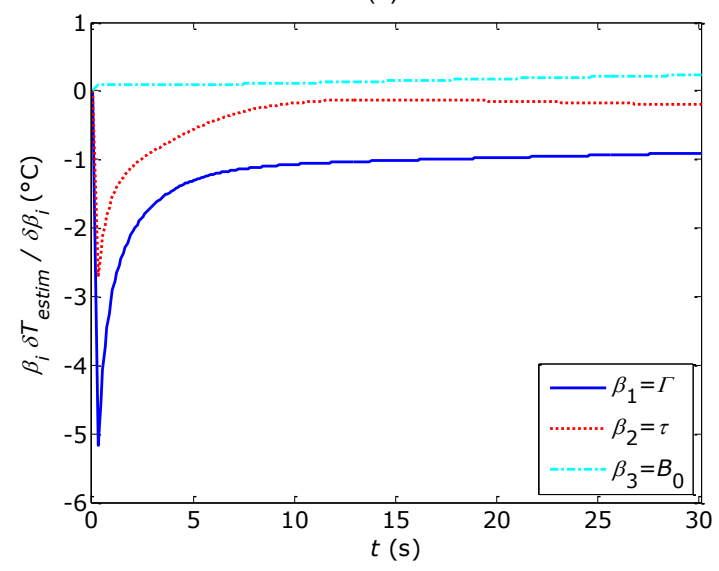

(c)

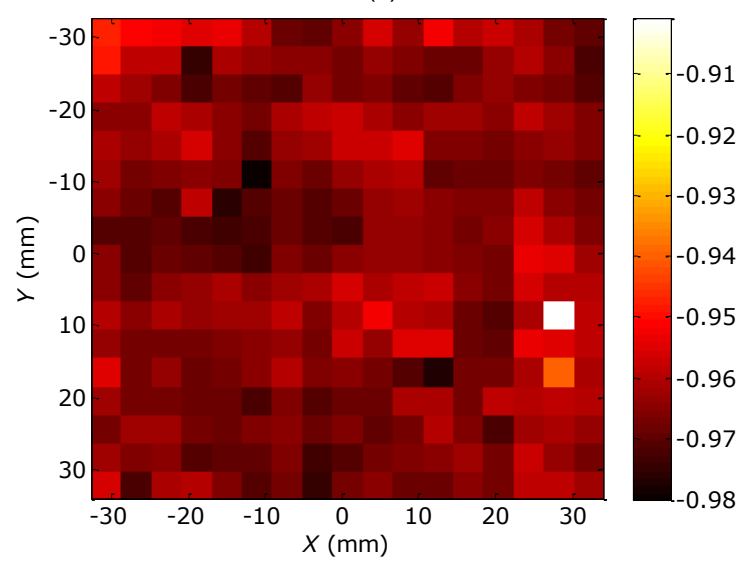

(b)

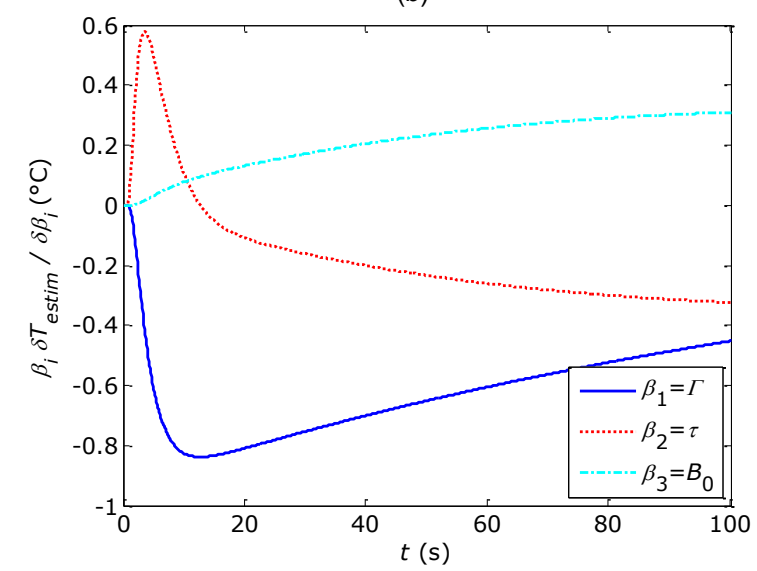

(d)

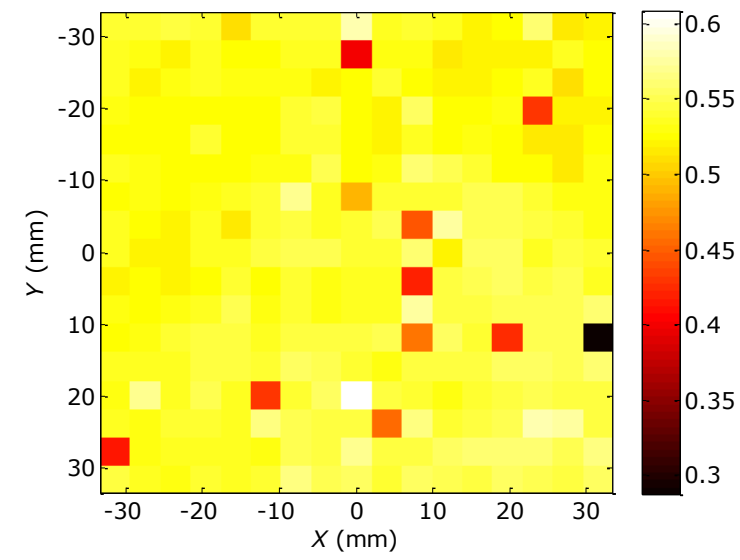

Fig. 3. Sensitivity study: (a) reduced sensitivity coefficients $C_{i}$ for front face measurements, (b) reduced sensitivity coefficients $C_{i}$ for rear face measurements, (c) correlation coefficient $\sigma_{\Gamma \tau}$ distribution for front face measurements, $(d)$ correlation coefficient $\sigma_{\Gamma \tau}$ distribution for rear face measurements

\subsection{Calibration procedure}

A calibration step is required in order to characterize the samples in terms of thermal diffusivity a and conductivity $k$. This procedure consists in replacing the studied sample by a material of known thermophysical properties, a PVC plate of thermal diffusivity $a=1.49 \times 10^{-7} \mathrm{~m}^{2} \cdot \mathrm{s}^{-1}$ and conductivity $\mathrm{k}=0.16 \mathrm{~W} \cdot \mathrm{m}^{-1} \cdot \mathrm{K}^{-1}$. These values were obtained using an independent experimental device [16]. This plate presents the same dimensions as the mosaic of the nine composite samples and is coated by a Nextel ${ }^{\mathrm{TM}}$ Velvet 811-21 paint of known optical properties: its infrared emissivity and visible absorptivity are equal to 0.97 . The plot of the measured and estimated temperatures, respectively $\Delta T_{\text {meas }}$ and $\Delta T_{\text {estim }}$ in the case of a front face experiment in the center of the plate and the residuals $\Delta T_{\text {meas }}-\Delta T_{\text {estim }}$ are presented in figure 4. This allows obtaining the estimated parameters in Eq. (7) and consequently the $W$ value if $a$ and $k$ are known.

The mean $W$ value is computed along the nine regions occupied by the samples. The obtained values for the different samples vary between 4139 and $5282 \mathrm{~J} . \mathrm{m}^{-2}$. At this stage, $W$ values correspond to energy density absorbed by a coating of absorption coefficient equal to $\alpha=0.97$. In order to obtain $W$ values for each composite sample, we must take into account the absorption coefficient $\alpha$ of each sample. These values were obtained independently using a home-made portable reflectometer operating in the visible spectral range. Values of $\alpha$ parameter ranging from 0.80 to 0.87 were obtained for the nine composite samples. After this calibration step, it is now possible to obtain the values of local thermal diffusivity $a$ and conductivity $k$ for each composite sample by using Eqs. 3(a) and 3(b). 
(a)

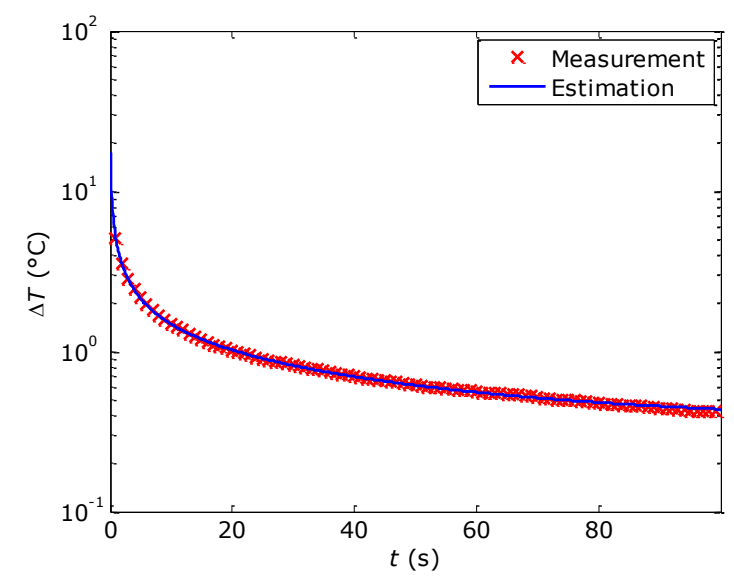

(b)

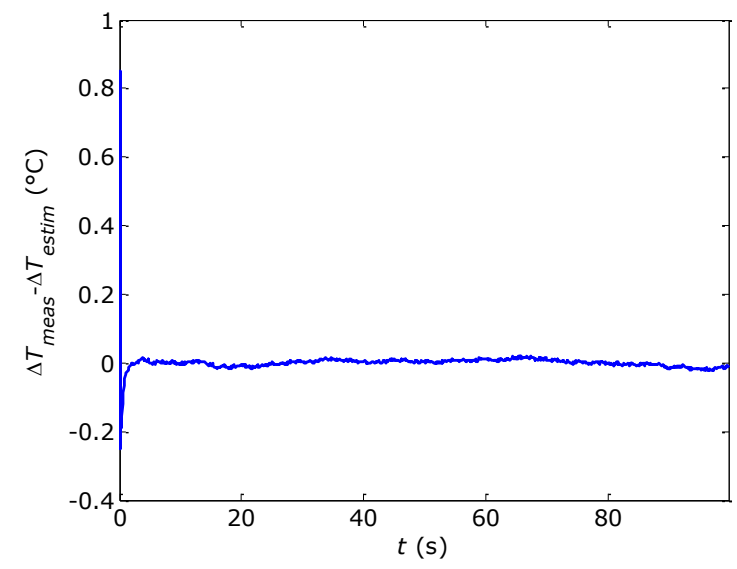

Fig. 4. Estimation results for a PVC plate and front face measurements: (a) measured and estimated temperatures, (b) temperature residuals

\subsection{Detailed estimation results obtained for a single sample}

In this section, estimation results are computed by the inverse procedure described in 3.2 for the sample located in the center of the mosaic. In particular, influences of the experiment type (front or rear face) and of the heat transfer model used are investigated.

Estimations are processed for the first accuracy model level (without heat exchange on each side of the sample). Measured and estimated temperature evolutions for front and rear faces experiments are presented in figure 5. We can observe that the experimental and theoretical curves do not match at the end of the experiment. This accuracy model does not allow modeling correctly the heat transfer occurred in the sample.

Then the influence of the heat exchange is examined for the case of an equivalent Biot number $B_{0}=B_{e}$ on both sides of the sample. As we can see in figure 6 measured and estimated temperature evolutions are in good accordance for both front and rear face experiments. Thermal diffusivity and conductivity distributions shown in figure 7 enable to visualize non negligible change of the thermophysical properties along the sample, probably due to porosity or fiber concentration variations inside the sample. Also the corresponding uncertainties are lower and less dispersed for the rear face experiment. These results and those resulting from the sensitivity study lead us to favor rear face estimations.

(a)

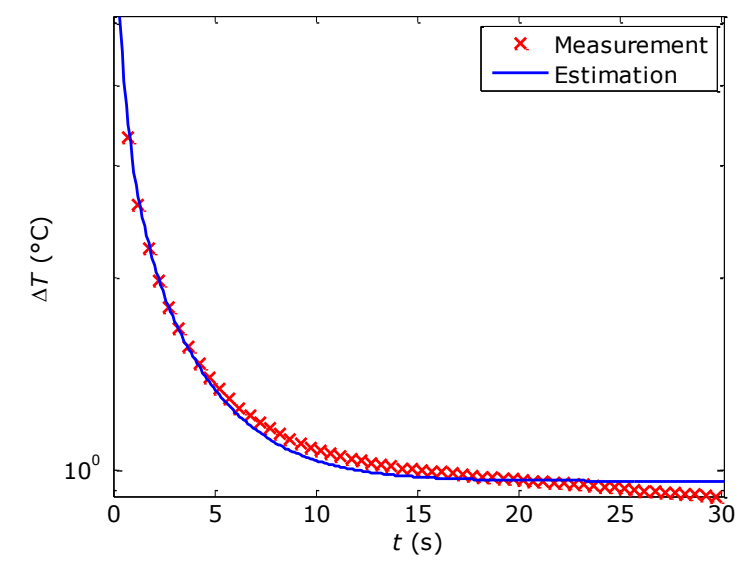

(b)

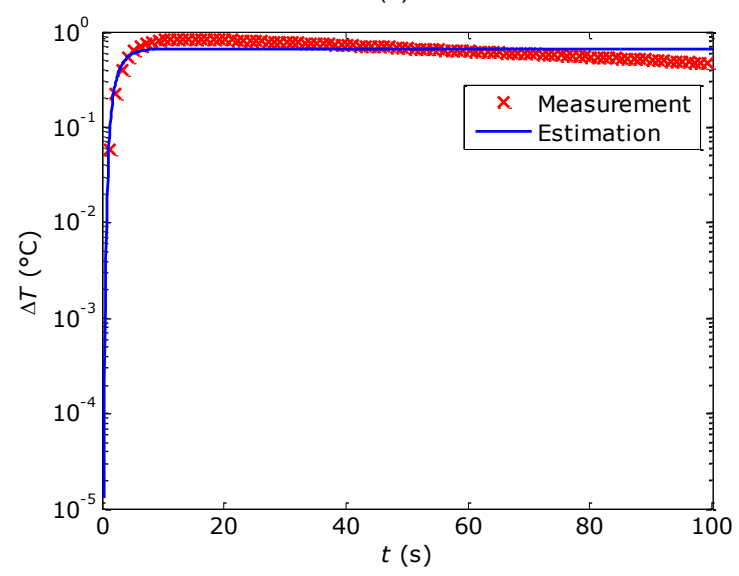

Fig. 5. Measured and estimated temperatures for the sample located at the center of the mosaic without heat exchange: (a) front face experiment, (b) rear face experiment 

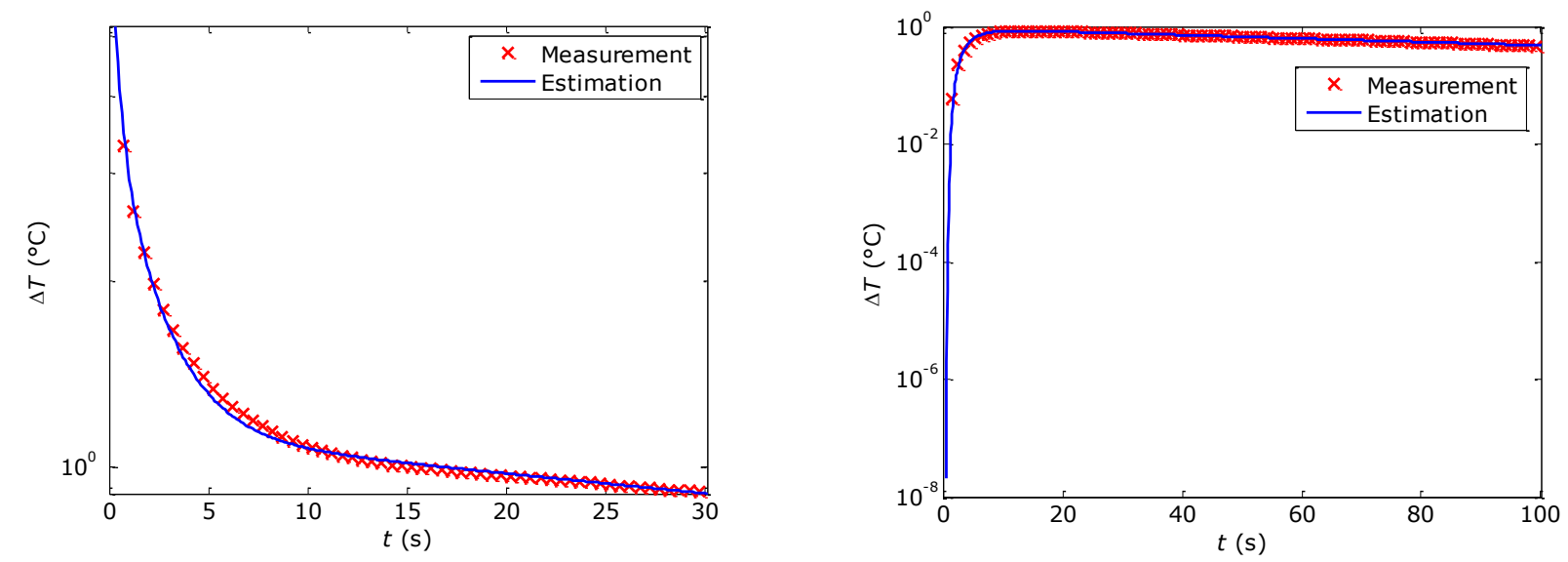

Fig. 6. Measured and estimated temperatures for the sample located at the center of the mosaic with equivalent heat exchange on both sides of the sample: (a) front face experiment, (b) rear face experiment

(a)
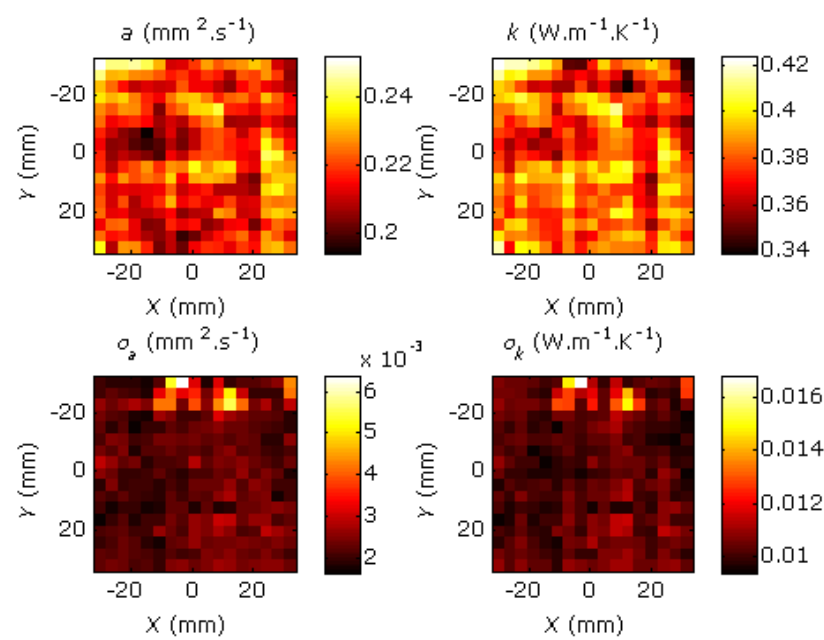

$o_{k}\left(\mathrm{~W} \cdot \mathrm{m}^{-1} \cdot \mathrm{K}^{-1}\right)$

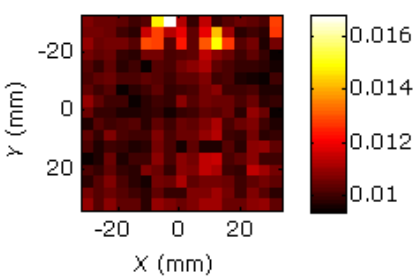

(b)
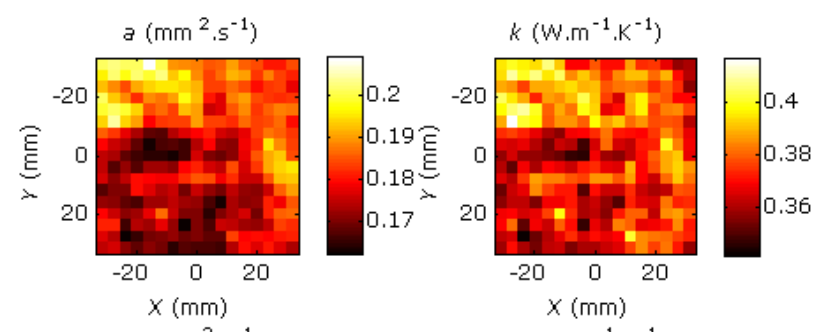

$o_{a}\left(\mathrm{~mm}^{2} \cdot \mathrm{s}^{-1}\right)$

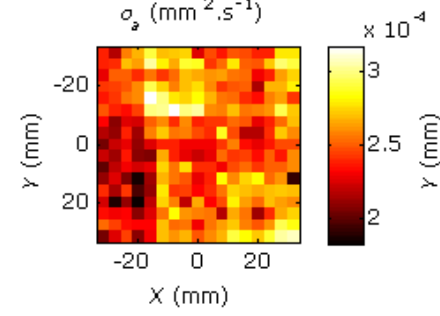

$o_{k}\left(\mathrm{~W} \cdot \mathrm{m}^{-1} \cdot \mathrm{K}^{-1}\right)$ $\times 10^{-3}$

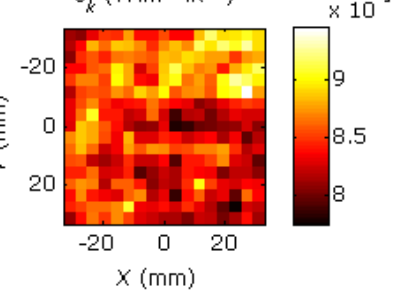

Fig. 7. Estimated thermal diffusivity and conductivity distributions and uncertainties along the sample located at the center of the mosaic with equivalent heat exchange on both sides of the sample: (a) front face experiment, (b) rear face experiment

\subsection{Estimation results summary}

Figure 8 shows a set of estimation results for the investigated samples by analyzing rear face measurements. Estimated parameters are given along with their minimum, mean and maximum values for each sample of the mosaic. These estimations lead to quite similar mean thermal diffusivity values. However parameter ranges can slightly differ between samples. Comparison of thermal diffusivity ranges with porosity rates measured by tomography was performed for a few samples.

Thermal diffusivity bar charts for samples 3, 4, 5 and 6 are presented in figure 9 along with known porosity rates. Two different behaviors can be observed depending on the porosity rates. On one hand, the mean diffusivity of the sample increases with porosity rate. On the other hand, the increase of the porosity rate tends to increase the dispersion of the measured thermal diffusivity. So measured thermal diffusivity seems closely linked to the sample porosity rate. 
(a)

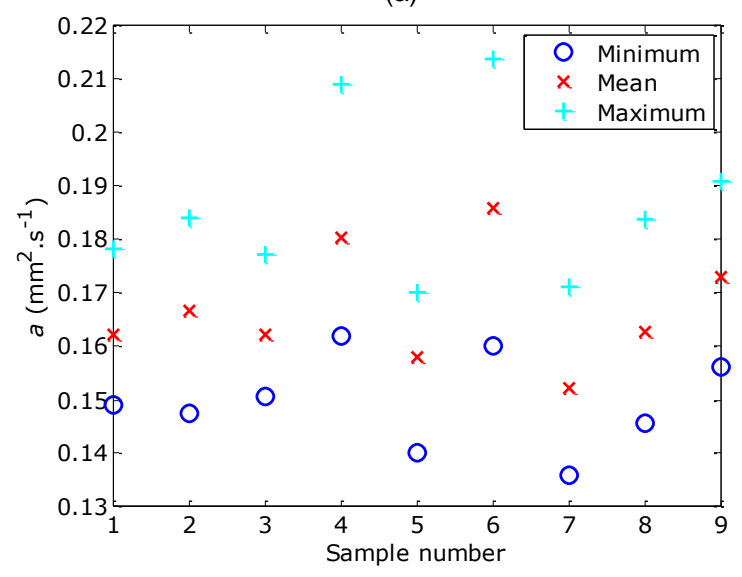

(b)

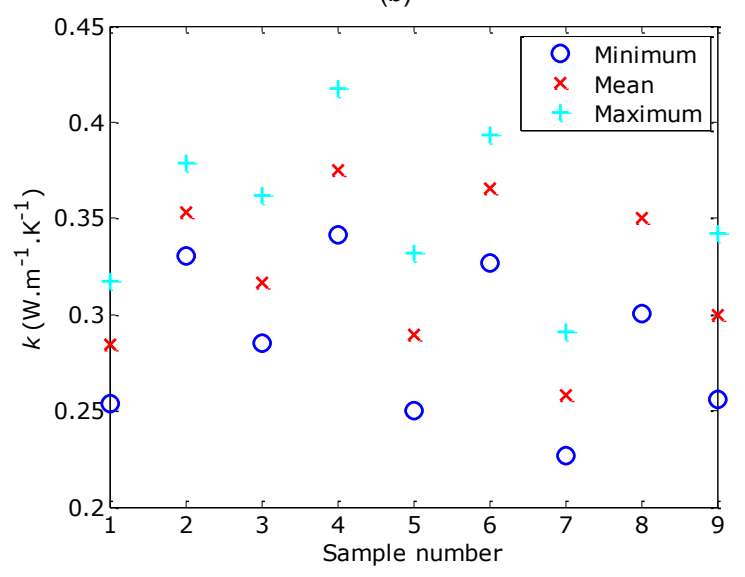

Fig. 8. Set of estimation results for the nine studied samples (rear face experiments): (a) estimated thermal diffusivity, (b) estimated thermal conductivity

Sample 3 - porosity rate $=6 \%$

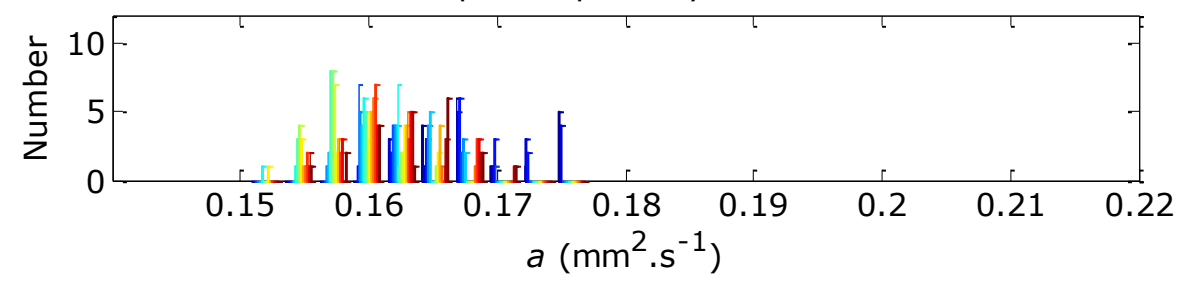

Sample 4 - porosity rate $=12 \%$

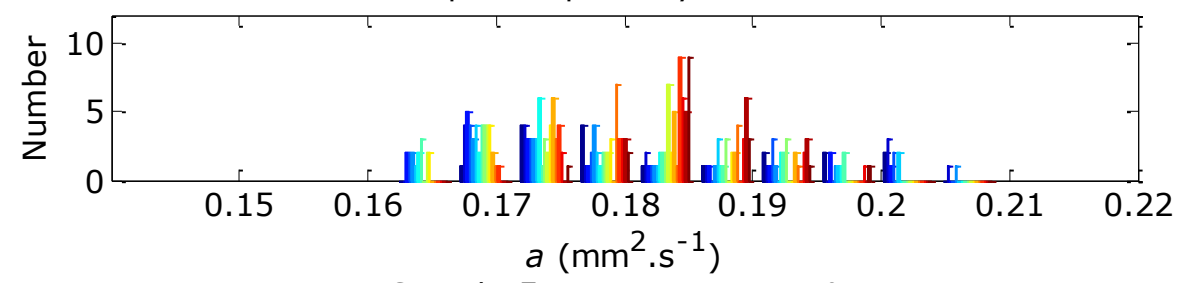

Sample 5 - porosity rate $=7 \%$

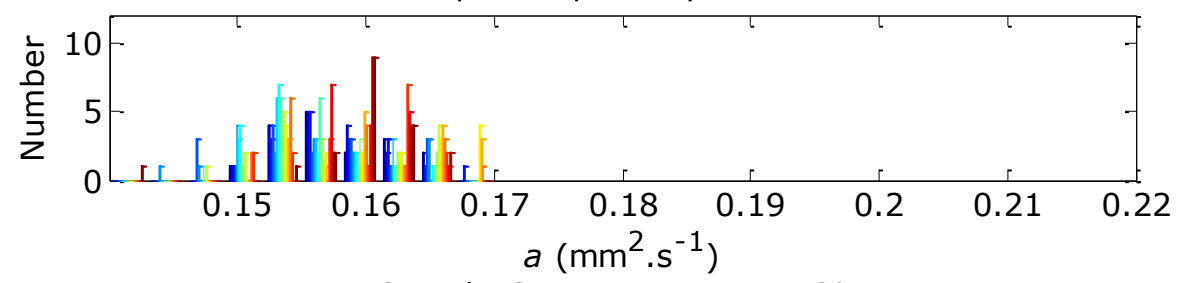

Sample 6 - porosity rate $=12 \%$

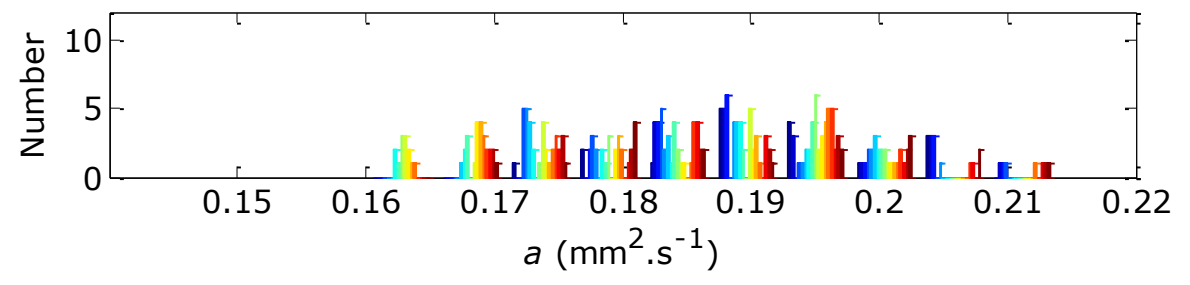

Fig. 9. Estimated thermal diffusivity bar charts for samples 3, 4, 5 and 6 (rear face experiments) 


\section{Conclusion}

Pulsed thermography coupled with detailed heat transfer modeling allowed investigating flax/epoxy composite samples presenting various porosity rates. Traditionally, porosity rates of this type of materials are evaluated by using ultrasonic method but uncertainties remain non negligible (around 25\%). The present study dealt with an active infrared thermography approach in order to link thermophysical properties to porosity rates.

The combination of a heat transfer modeling based on integral transform technique and an inverse procedure lead to estimate different parameters characterizing the thermal behavior of the studied sample. A calibration procedure using a sample of known thermophysical properties allowed estimating spatial thermal diffusivity and conductivity distributions for each sample. Sensitivity study and estimation results showed that rear face measurements are more suitable than front face measurements to characterize the sample. Finally it appeared that thermal diffusivity distributions in terms of mean value and dispersion along the sample could provide interesting information about the sample porosity rate. On one hand, the more the porosity rate is, the more the mean diffusivity of the sample is. On the other hand, the increase of the porosity rate tends to increase the dispersion of the estimated thermal diffusivity.

\section{REFERENCES}

[1] Birt, E., A, and R. Smith, A, "A review of NDE methods for porosity measurement in fibre-reinforced polymer compoistes". Insight, vol.46 (11), pp. 681-686, 2004.

[2] Schuller, J. and Oster, R., "Classification of porosity by ultrasonic carbon fibre helicopter structures based on micro-computed tomography" European conference on non-destructive testing Proceedings.Berlin (Germany), pp. 25-29, 2006.

[3] Ledru, Y., " Etude de la pororsité dans les matériaux composites stratifiés aéronautiques", PhD Thesis Université de Toulouse (France), 2009

[4] Ledru, Y., et al. "Quantification 2-D et 3-D de la porosité par analyse d'image dans les matériaux stratifiés aéronautiques". Comptes Rendus des JNC 16, Toulouse, 2009.

[5] Kastner, J., et al., "Defect and porosity determination of fibre reinforced polymers by X-ray computed tomography". $2^{\text {nd }}$ International Symposium on NDT in Aerospace, pp.1-12, 2010.

[6] Chu, T., Russell, S.S. and Wlaker J.L., "Porosity measurement in laminate composites by thermography and FEA" SEM Annual Conference Proceedings, 2001.

[7] Mayr, G. and Hendorfer, G. "Porosity determination by Pulsed thermography in reflection mode", Proceedings of $10^{\text {th }}$ Quantitative InfraRed Thermography conference, paper QIRT2010-933. Québec (Canada), 2010.

[8] Connoly, M.P., "The measurement of porosity in composite materials using infrared thermography". Journal of reinforced plastics and composites". vol. 11 (12): pp. 1367-1375, 1992.

[9] Zalameda, J.N. and Winfree W.P., "Quantitative thermal diffusivity measurements on composite fiber volume fraction" Review of progress in quantitative non-destructive evaluation Proceedings, Thompson D.O. and Chimenti D.E. ed, pp. 1289-1295, 1989.

[10] M. Assarar et al., "Influence of water ageing on mechanical properties and damage events of two reinforced composite materials: Flax-fibres and glass-fibres". Materials \& Design, vol.32 (2), pp. 788-795, 2011.

[11] Vavilov V., Transient thermal NDT: conception in formulae, QIRT 1992, Paris (France), pp. 229-234, 1992.

[12] Tsacalos D., "Résolution des problèmes de thermocinétique linéaire par la méthode des transformations intégrales finies". Revue Générale de Thermique, vol. 214, pp. 609-619, 1979.

[13] Marquardt D.W., "An algorithm for the least squares estimation of non linear parameters". Journal of Society for Industrial and Applied Mathematics, vol. 1, pp. 431-441, 1963.

[14] Söderströn T, Stoica P., "System Identification”. Englewood Cliffs, NJ:: Prentice-Hall, pp. 33, 1989.

[15] Huang C.H., özisik M.N., "Direct integration approach for simultaneously estimating temperature dependent thermal conductivity and heat capacity". Numerical Heat Transfert Part A, vol. 20, pp. 95-110, 1991.

[16] Boudenne A., Ibos L., Géhin E., Candau Y., "A simultaneous characterisation of thermal conductivity and diffusivity of polymer materials by a periodic method". Journal of Physics D : Applied Physics, vol. 37 (1), pp. 132-139, 2004. 